\title{
Repopulation of Tumour Cells During Radiotherapy is Doubled During Treatment Gaps
}

\author{
RAFAL TARNAWSKI*, KRZYSZTOF SKLADOWSKI, ANDRZEJ SWIERNIAKa ${ }^{\mathrm{a}}$, ANDRZEJ WYGODA and \\ ANNA MUCHA
}

Centre of Oncology Maria Sklodowska-Curie Memorial Institute branch in Gliwice Wyb. Armii Krajowej 15, 44-101 Gliwice Poland; ${ }^{a}$ Department of Automatic Control, Silesian Technical University ul. Akademicka 16, 44-100 Gliwice Poland

(Received 24 November 1998; In final form 9 August 1999)

\begin{abstract}
The aim of this work is to analyse the proliferation of tumour cells in the treatment gap during the radiotherapy for head neck cancer.

\section{Material and Methods}

The clinical material is based on records of head and neck patients treated by radiotherapy alone in our institution. The effect of radiotherapy was assumed to be described by a linearquadratic model. The patient data were fitted directly to the radiobiological model and the parameters were estimated using maximum-likelihood procedures.

\section{Results}

According to our model results of treatment were significantly correlated with Normalised Total Dose of radiation, the tumour progression (according to TNM), the overall treatment time and the gap duration. The laryngeal cancers had better prognosis then cancers of oroand nasopharynx. When the treatment time is prolonged without treatment interruptions 0.36 Gy/day is lost due to the repopulation of tumour cells. During the treatment gap proliferation is faster and $0.67 \mathrm{~Gy} /$ day is lost.
\end{abstract}

\section{Conclusion}

Proliferation of tumour cells is faster during the treatment gap then during the days with irradiation.

Keywords: radiotherapy, treatment time, treatment gaps, Tumor proliferation

\section{BACKGROUND}

The aim of radiation therapy (RT) is to deliver a dose of radiation to a tumour volume with as minimal damage as possible to the surrounding normal tissue resulting ideally in tumour cure, prolongation of survival, with high quality of life standard. Late reacting normal tissues limit the dose of ionising radiation delivered during RT.

Head and neck cancers are readily cured by RT during the early stages of tumour growth. Local regional eradication of head and neck cancer is the most important for prolongation of survival as metastases are very rare.

\footnotetext{
${ }^{*}$ Corresponding author.
} 


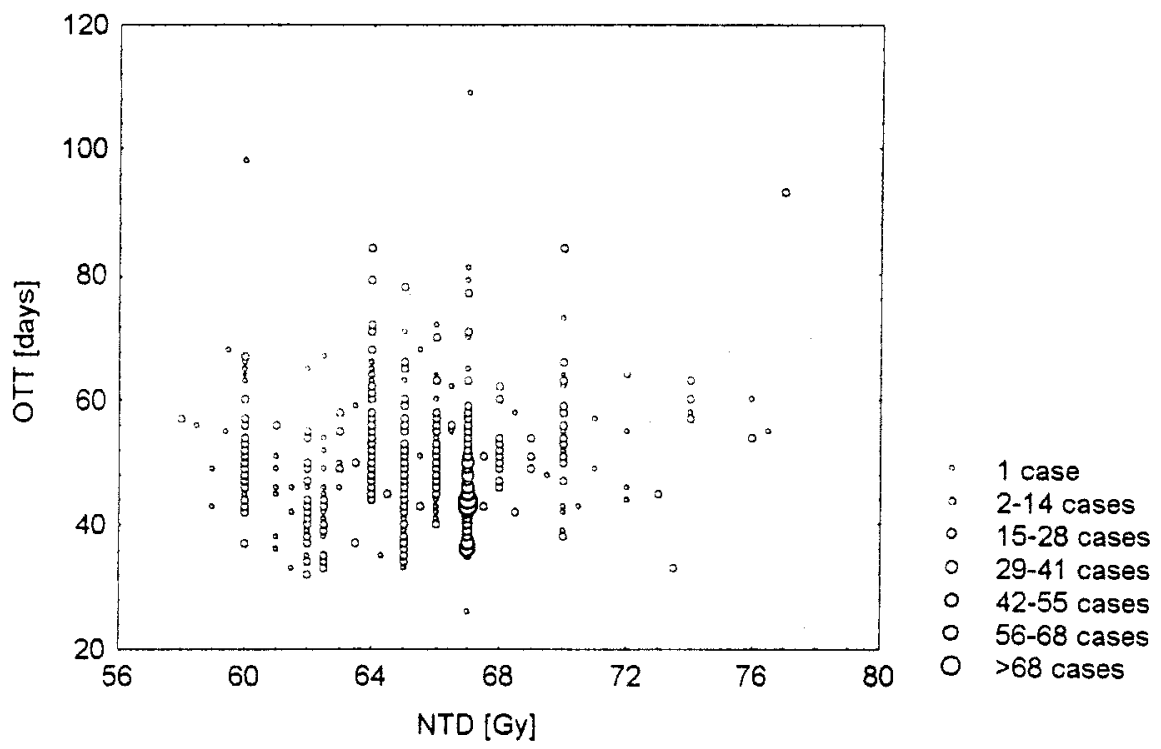

FIGURE 1 The distribution of the normalised total doses (NTD) of radiation together with overall treatment time (OTT) and the number of patients.

Both retrospective and some prospective randomised clinical studies showed the importance of treatment time for local control of disease [1-3]. Although the clinical observation of the growth of unirradiated cancers showed the relatively long doubling times of tumour volumes (median 30 days), many studies showed that the prolongation of the overall treatment time resulted in worse results of radiation therapy, and the doubling time of tumour cells might be reduced during RT to 5 days. The absolute measurements of proliferation during RT are very difficult as after first doses of radiation the majority of tumour cells are inactivated, and measured mitoses reflects mainly abortive divisions. The only possibility to estimate repopulation of tumour cells is measurements of radiation dose required to balance proliferation during one day prolongation of treatment [4].

The aim of this work is to analyse the proliferation of tumour cells in the treatment gap during the radiotherapy for head and neck cancer.

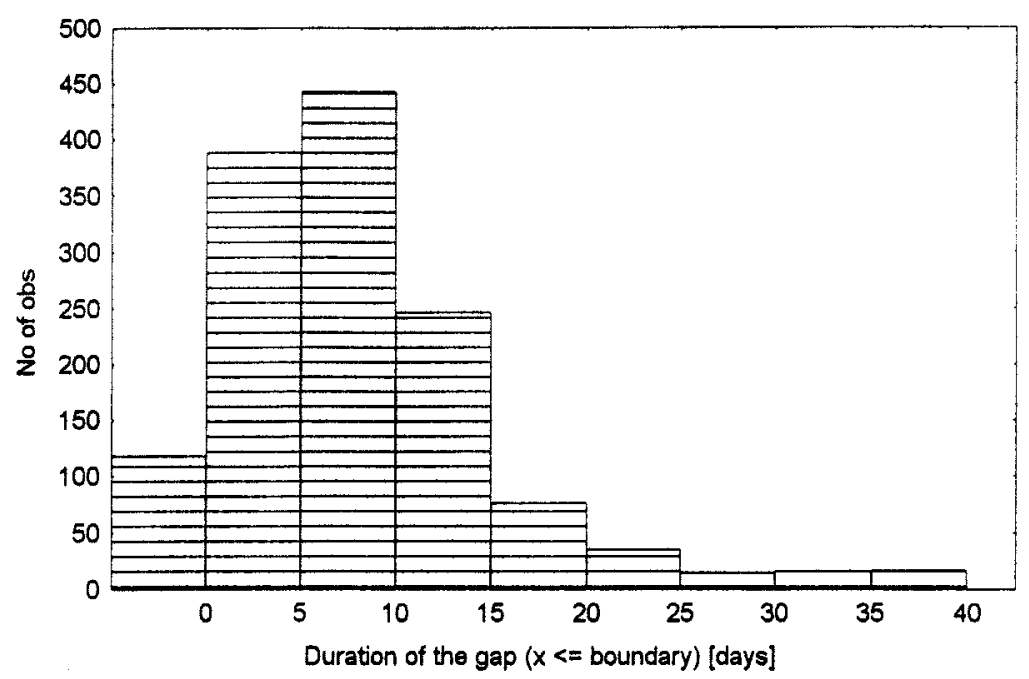

FIGURE 2 The cumulative duration of all gaps for the whole group of patients. 
TABLE I The distribution of tumour and nodal stage according to the TNM classification for different tumour localisation

\begin{tabular}{|c|c|c|c|c|c|}
\hline Oral pharynx & $\mathrm{N}_{0}$ & $\mathrm{~N}_{1}$ & $\mathrm{~N}_{2}$ & $\mathrm{~N}_{3}$ & Row Totals \\
\hline $\mathrm{T}_{1}$ & 19 & 6 & 2 & 2 & 29 \\
\hline $\mathrm{T}_{2}$ & 68 & 26 & 35 & 6 & 135 \\
\hline $\mathrm{T}_{3}$ & 40 & 29 & 44 & 9 & 122 \\
\hline $\mathrm{T}_{4}$ & 3 & 4 & 3 & 2 & 12 \\
\hline Total & 130 & 65 & 84 & 19 & 298 \\
\hline Nasopharynx & $\mathrm{N}_{0}$ & $\mathrm{~N}_{1}$ & $\mathrm{~N}_{2}$ & $\mathrm{~N}_{3}$ & \\
\hline $\mathrm{T}_{1}$ & 2 & 1 & 1 & 1 & 5 \\
\hline $\mathrm{T}_{2}$ & 5 & 3 & 4 & 7 & 19 \\
\hline $\mathrm{T}_{3}$ & 3 & 3 & 5 & 3 & 14 \\
\hline $\mathrm{T}_{4}$ & 4 & 0 & 1 & 0 & 5 \\
\hline Total & 14 & 7 & 11 & 11 & 43 \\
\hline Larynx & $\mathrm{N}_{0}$ & $\mathrm{~N}_{1}$ & $\mathrm{~N}_{2}$ & $\mathrm{~N}_{3}$ & \\
\hline $\mathrm{T}_{1}$ & 54 & 10 & 3 & 2 & 69 \\
\hline $\mathrm{T}_{2}$ & 229 & 53 & 44 & 8 & 334 \\
\hline $\mathrm{T}_{3}$ & 212 & 88 & 76 & 19 & 395 \\
\hline $\mathbf{T}_{4}$ & 112 & 50 & 43 & 6 & 211 \\
\hline Total & 607 & 201 & 166 & 35 & 1012 \\
\hline Column Total & 751 & 273 & 261 & 65 & 1350 \\
\hline
\end{tabular}

\section{MATERIALS AND METHODS}

The clinical material is based on the records of 1350 patients treated by radiotherapy alone in Centre of Oncology Maria Sklodowska-Curie Memorial Institute in Gliwice during the period between 1980-89. All patients had histologically confirmed squamous cell carcinoma of larynx or pharynx (oral pharynx or nasopharynx), no prior therapy, nodal stage $\mathrm{N}_{0-3}$, no distant metastases and good performance status (Karnofsky index above 70). Patients' data consisted of the set of the prognostic factors such as tumour localisation, tumour stage, nodal stage, and the observation of tumour local control after treatment given as the time to recurrence or recurrence free observation time.

All patients were irradiated using a cobalt unit and a technique that employed two lateral, opposed fields. This was performed once a day, 5 times a week with no treatment on during weekends. The dose was calculated in the mid-plane on the axis of the beam. The fractionation pattern was described in details elsewhere [5]. Figure 1 shows the distribution of the total doses (normalised to NTD) of radiation together with the overall treatment time (OTT) and the number of patients. The patients' characteristics namely tumour localisation, tumour and nodal stage (TNM classification-UICC 1978) are given in Table I.

The gaps during the treatment were of different origin. Some gaps were related to the machine breaks, holidays, and problems with transportation of patients. The other group of treatment interruptions was related to severe mucosal reactions or intercurrent disease. A small group of patients was treated with a planned gap (split) in the middle to reduce the early mucosal radiation damage. Some patients had more than one treatment interruption. The cumulative duration of all gaps for the whole group of patients is given in Figure 2.

\section{MATHEMATICAL MODELS}

The effect of radiotherapy was assumed to be described by a linear quadratic model. The local control was defined, as the case of no clinical evidence of tumour, or if recurrence was suspected but biopsy was negative, the case of no pathologic evidence of tumour. 
It was assumed that the tumour is cured when all its cells are inactivated. The expected number of cells after radiotherapy was calculated by multiplying the initial number of cells by survival fraction. The most of clinical data show that the survival fraction (SF) is the linear-quadratic function of a dose per fraction $\left(\mathrm{d}_{-} \mathrm{fx}\right)$.

$$
\mathrm{SF}=\exp \left(-\alpha \mathrm{d}_{-} \mathrm{fx}-\beta \mathrm{d}_{-} \mathrm{fx}^{2}\right)
$$

The survival fraction after $\mathrm{n}$ fractions and after all the total dose of $T D=n d_{-} f x$ is

$$
\mathrm{SF}=\exp \left(-\alpha \mathrm{TD}-\beta \mathrm{TD} \mathrm{d}_{-} \mathrm{fx}\right)
$$

It was not possible to estimate radiosensitivity $\alpha$ and fractional sensitivity $\alpha / \beta$ parameters from our data because of limited range of fractional doses, so the value of $\alpha / \beta$ was fixed to $15 \mathrm{~Gy}$, as estimated for squamous cell carcinomas of head and neck [6]. The value of $\alpha / \beta=15$ Gy was chosen because the majority of clinical and experimental data show that for human squamous cell carcinomas the most probable $\alpha / \beta$ value is in the range of $10-20 \mathrm{~Gy}$. In order to allow the simple presentation of data Normalised Total Dose (NTD) [6] was calculated from the equation:

$$
\mathrm{NTD}=\mathrm{TD}\left(\alpha / \beta+\mathrm{d}_{-} \mathrm{fx}\right) /(\alpha / \beta+2)
$$

where TD denotes physical dose. NTD is the dose given in standard fractionation using the dose per fraction of $2 \mathrm{~Gy}$, which has the same survival fraction as the dose given with non-standard dose per fraction $\left(d_{-} f x\right)[6]$.

It was assumed that the tumour proliferation could be described by a simple exponential growth with the growth constant $\lambda$. The dose to compensate one additional day of treatment was calculated as $\lambda / \alpha$, while the dose for gaps was defined as $\delta / \alpha$. Therefore one could treat $\delta / \lambda$ as a scaling factor for gaps.

Since it was not possible to estimate the volumes of tumours from the retrospective data, tumour stage groups according to TNM classification were used in analysis.

The patient data were fitted directly to the radiobiological model describing Tumour Control Probability (TCP) given by the formula:

$$
\begin{aligned}
\mathrm{TCP}= & \exp (-\exp (\ln \mathrm{K}-\alpha \mathrm{NTD} \\
& +\lambda \mathrm{OTT}+\delta \text { gap_duration }))
\end{aligned}
$$

The parameters were estimated using maximumlikelihood procedures with the help of the BMDP statistical software, which computes the maximum likelihood estimates of a user stated density function F via the Newton-Raphson iterative method [7]. The TCP model is based on the assumption that the local control is achieved when all the functionally clonogenic cells are inactivated. The parameter $\ln \mathrm{K}$ is the natural logarithm of the initial number of clonogenic cells. This number is increased or decreased by the tumour stage or the tumour localisation. The overall treatment time (OTT) and the gap duration are expressed in days. The parameter $\alpha$ reflects the radiosensitivity of the tumour cells, and the survival fraction $(\mathrm{SF})$ is defined as:

$$
\mathrm{SF}=\exp (-\alpha \mathrm{NTD})
$$

The expected number of cells after radiotherapy is given as:

$$
\mathrm{N}=\mathrm{K} \mathrm{C} \mathrm{SF}
$$

where constant $\mathrm{C}$ is different for different tumour stages and localisation. The constant $C$ is the parameter introduced in order to take into account the most important prognostic factors such as tumour localisation and clinical tumour stage related to the tumour volume, local progression (T-stage), and involvement of lymph nodes ( $\mathrm{N}$-stage).

The tumour stage or the tumour localisation have been introduced to Equation (4) in the form of categorical variables and the estimates reflect the difference between the number of functionally clonogenic cells for the first stage of the disease and the other stages or between localisation in oral pharynx (for tumour localisation) and the other localisations. Thus the regression Equation (4) is treated as the basic one and is relevant to the oral cavity in the $T_{1}$ tumour and the $\mathrm{N}_{0}$ nodal stage. For the laryngeal and nasopharyngeal cancers we have introduced a correction term $c_{t}$ which estimates are given in Table II as Larynx Est. and Nasophar.Est respectively. Similarly we introduce parameters $T_{i}$ and $N_{j}(i=2,3,4 ; j=1,2,3)$ for different tumour and nodal stages the estimates of 
TABLE II The maximum-likelihood estimates of mixture model parameters as described in the Material and Method section. The maximum likelihood ratio test was used to yield p-values obtained from the forward stepwise regression and the maximum likelihood ratio test. The base line stratum was the Weibull distribution of the time to recurrence

\begin{tabular}{|c|c|c|c|c|}
\hline Parameter & Estimate & S.E. & $\begin{array}{l}95 \% \text { conf. } \\
\text { interval. }\end{array}$ & p-value \\
\hline $\ln K$ & 1.88 & 1.30 & $-0.509,4.47$ & $\mathrm{p}=0.14$ \\
\hline Nasophar.Est & -0.286 & 0.250 & $-0.706,0.12$ & $p=0.24$ \\
\hline Larynx Est. & -0.508 & 0.114 & $-0.734,-0.284$ & $\mathrm{p}<0.0001$ \\
\hline $\mathrm{T}_{2}$ Est. & 1.83 & 0.313 & $1.23,2.50$ & $\mathrm{p}<0.0001$ \\
\hline$T_{3}$ Est. & 2.74 & 0.314 & $2.14,3.42$ & $\mathrm{p}<0.0001$ \\
\hline $\mathrm{T}_{4}$ Est. & 2.84 & 0.322 & $2.22,3.53$ & $\mathrm{p}<0.0001$ \\
\hline $\mathrm{N}_{1}$ Est. & 0.311 & 0.111 & $0.092,0.527$ & $\mathrm{p}=0.005$ \\
\hline $\mathrm{N}_{2}$ Est. & 0.721 & 0.119 & $0.463,0.956$ & $\mathrm{p}<0.0001$ \\
\hline $\mathrm{N}_{3}$ Est. & not calculated & did not reach & convergence & \\
\hline$\alpha$ (radiosensitivity) $[1 / \mathrm{Gy}]$ & 0.0846 & 0.0186 & $0.0474,0.121$ & $\mathrm{p}<0.0001$ \\
\hline$\lambda$ (time correction) $[1 /$ day $]$ & 0.0304 & 0.00898 & $0.0129,0.0480$ & $p=0.0007$ \\
\hline$\delta$ (time correction) $[1 /$ day $]$ & 0.0266 & 0.0112 & $0.0060,0.0486$ & $\mathrm{p}=0.008$ \\
\hline l (Weibull distrib.) & 0.276 & 0.0157 & $0.238,0.308$ & \\
\hline th (Weibull distrib.) & 0.867 & 0.0230 & $0.812,0.912$ & \\
\hline
\end{tabular}

which are also presented in Table II as $T_{i}$ Est. and $\mathrm{N}_{\mathrm{j}}$ Est. respectively.

Thus the radiobiological model may be given in the following form:

$$
\begin{aligned}
\mathrm{TCP}= & \exp (-\exp (\ln \mathrm{K}-\alpha \mathrm{NTD}+\lambda \mathrm{OTT} \\
& \left.\left.+\delta \text { gap_duration }+\mathrm{c}_{\mathrm{t}}+\mathrm{T}_{\mathrm{i}}+\mathrm{N}_{\mathrm{j}}\right)\right)
\end{aligned}
$$

where:

$$
\begin{aligned}
& c_{t}= \begin{cases}0 & \text { for oral pharynx } \\
\text { LarynxEst. } & \text { for larynx } \\
\text { Nasophar.Est } & \text { for nasopharynx }\end{cases} \\
& T_{i}= \begin{cases}0 & \text { for } T_{1} \text { tumour stage } \\
T_{i} \text { Est. } & \text { for } T_{1} \text { stages }(i=2,3,4)\end{cases} \\
& N_{j}= \begin{cases}0 & \text { for } N_{0} \text { nodal stage } \\
N_{j} \text { Est. } & \text { for } N_{j} \text { stages }(j=1,2,3)\end{cases}
\end{aligned}
$$

Not all patients had complete observations, so the mixture model was used as proposed by Bentzen $e t$ al. $[8,9]$ in order to allow for non-zero cure rate. The mixture model consists of the Equation (7) with a Weibull distribution for the underlying (conditional upon ultimate failure) time to recurrence distribution. The forward-stepwise maximum-likelihood regression was used with the mixture model with binomial likelihood for binary data. The confidence intervals for the parameter estimates were calculated using the Cook and Weisberg method [10]. The likelihood-ratio test was used in order to test the improvement of fit, when the additional factors are introduced to the model [10].

\section{RESULTS}

The maximum-likelihood estimates of mixture model parameters are given in Table II.

According to the mixture model the results of treatment were significantly correlated with NTD, the tumour progression (according to TNM), the overall treatment time, and the gap duration. The laryngeal cancers had a better prognosis than the cancers of oro- and nasopharynx.

The TCP (Tumour Control Probability) for the $\mathrm{T}_{1}$ laryngeal cancer treated during a 45 days period without treatment breaks is shown in Figure 3. The results of the treatment with the conventional doses (NTD in the range of 60-66 Gy) are very good, about $90 \%$ of patients are permanently cured.

The results of the treatment of the laryngeal $\mathrm{T}_{3} \mathrm{~N}_{0}$ cancer treated during a 49 days period are shown in Figure 4. The results of the treatment with the standard 70 Gy are unsatisfactory with only about $50 \%$ of patients being permanently cured. The dose of radiation must not be increased because of the risk of severe normal tissue damage. The outcome of the treatment is even worse if the treatment time is prolonged either by increasing the number of fractions 


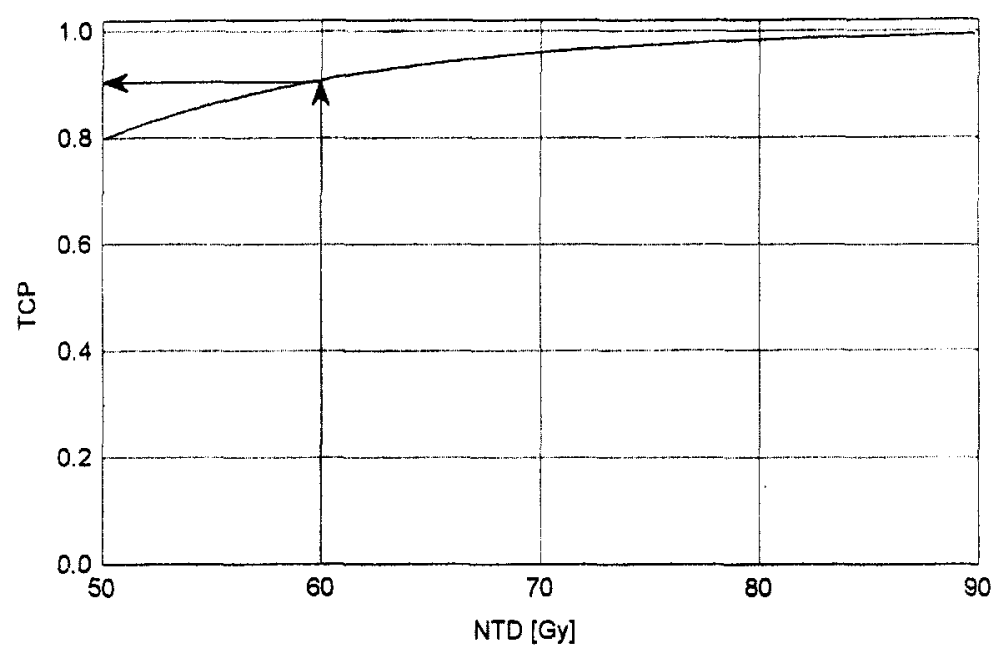

FIGURE 3 The predicted TCP function for patient with laryngeal cancer $\mathrm{T}_{1} \mathrm{~N}_{0}$ treated during a 45 days period without treatment gaps depending on the delivered normalised dose of radiation (NTD). The standard treatment is $60 \mathrm{~Gy}$.

(for instance decrease of dose per fraction from $2 \mathrm{~Gy}$ to $1.8 \mathrm{~Gy}$ in order to reduce the early normal tissue radiation damage) $-\lambda / \alpha=0.36$ Gy/day is lost, or by a break in the treatment. When the treatment is interrupted the drop in TCP is even greater as for one day prolongation of the treatment $\lambda / \alpha+\delta / \alpha=0.67$ Gy is lost due to the proliferation of the tumour cells.

Figure 5 shows the predicted TCP function for a patient with the laryngeal cancer $\mathrm{T}_{3} \mathrm{~N}_{0}$ treated with standard dose $70 \mathrm{~Gy}$. The fitted function displays the loss of TCP when the treatment time is prolonged either by decreasing the daily dose (no gaps) or by treatment interruptions. When the treatment gap is longer than 10 days the drop in TCP is larger then $20 \%$ (from $48 \%$ to $28 \%$ ). In our material about $30 \%$ (401/1350) of patients had treatment gaps of 10 days or longer.

\section{DISCUSSION}

The results of conventional irradiation are usually good for early stages of head and neck cancer. The

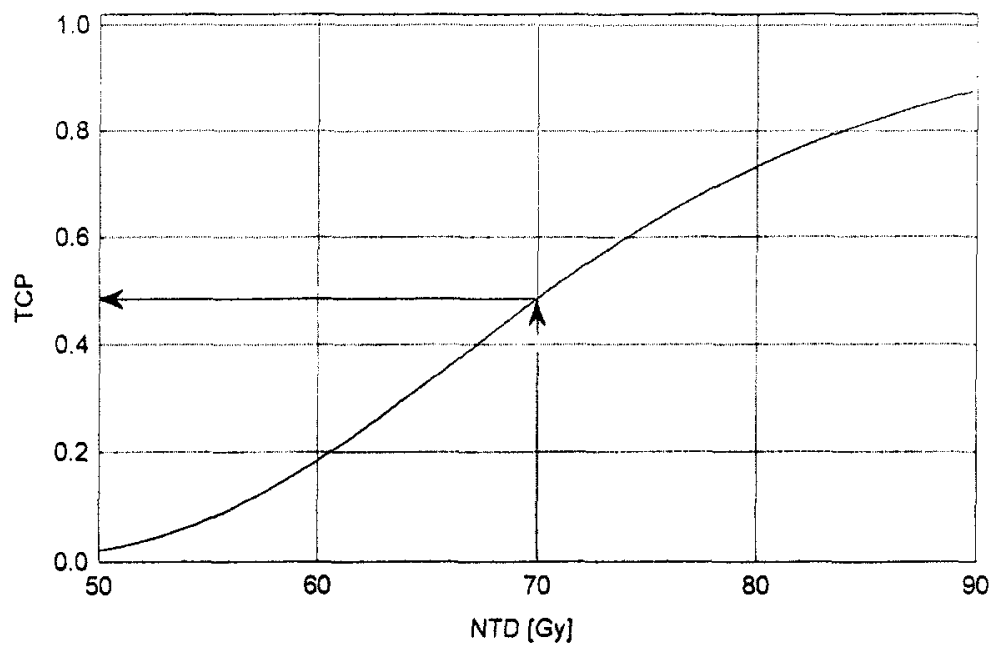

FIGURE 4 The predicted TCP function for a patient with the laryngeal cancer $\mathrm{T}_{3} \mathrm{~N}_{0}$ treated during a 49 days period without treatment gaps depending on the delivered normalised dose of radiation (NTD). The standard treatment is $70 \mathrm{~Gy}$. 


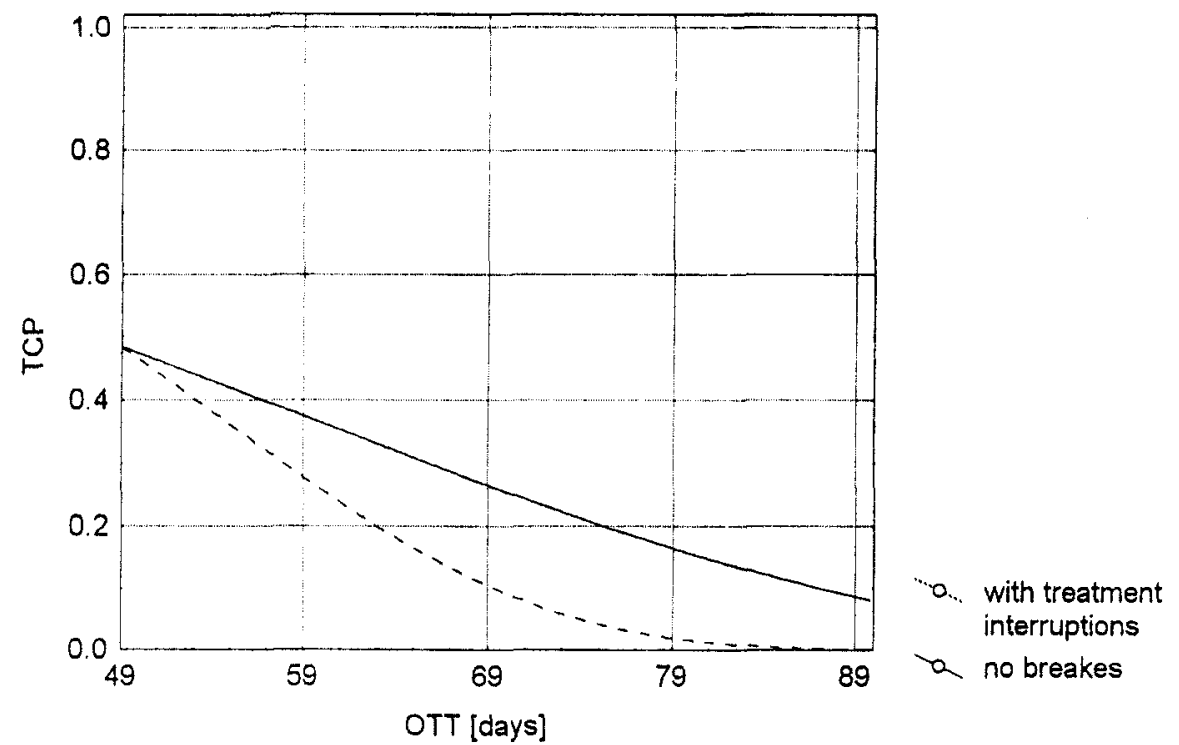

FIGURE 5 The predicted TCP function for a patient with the laryngeal cancer $\mathrm{T}_{3} \mathrm{~N}_{0}$ treated with the standard dose of 70 Gy. The fitted function displays the loss of TCP when the treatment time is prolonged either by decreasing the daily dose (no gaps) or by treatment interruptions.

conventional doses which do not induce severe normal tissue damage result in a very good tumour local control. In our data about $90 \%$ of $\mathrm{T}_{1}$ patients were cured, and this group of patients is not a candidate for the altered fractionation. However the outcome of radiotherapy is not so good for advanced stages of the disease. The combined treatment consisting of radical surgical treatment and post-operative irradiation is effective if the surgical resection is feasible. Radical operation is usually mutilating resulting in the loss of the organ function. In an attempt to increase the therapeutic ratio (increase in local control without inducing the late radiation damage), various fractionation schedules have been used in radiation therapy delivery.

The dose-time considerations lead to a complex function of the total dose, the time and the number of fractions related to a biological effect within a given tissue volume. This phenomenon is closely related to four radiobiological R's of radiation therapy: repair, repopulation, redistribution and reoxigenation [10]. The first two R's are the most important for the clinical practice.

The repair of radiation damage is reflected in the linear-quadratic part of the equation. The $\alpha / \beta$ parameter reflects the fractionation sensitivity. In general, the late reacting tissues are more sensitive to the increase of the fractional dose. The fractionation with small doses per fraction reduces the biological dose in the dose-limiting late reacting tissues, the effect of fractionation is not so pronounced in the rapidly growing tissues such as tumour cells. The Normalised Total Dose (NTD) or the Biologically Effective Dose (BED) are used in the clinical practice for calculation of the biological dose, and they are based on the linear-quadratic Equation [10]. Reducing the fractional size may allow the escalation of total dose but it results in the prolongation of the treatment.

The repopulation of tumour cells between fractions may limit the efficacy of radiation therapy when the treatment is prolonged. Usually a simple exponential growth of tumour cells is assumed [10]. Some authors claim that there is a lag period of 20-30 days after the start of the treatment before the accelerated proliferation begins [11]. As the overall treatment times of the patients used in our analysis are longer than 28 days we could not test this possibility in our analysis. Only data sets with treatment time shorter than 3-4 weeks showed the effect of the delay of accelerated proliferation [12]. 
Our study shows how devastating for the treatment outcome may be the prolongation of the treatment due to treatment gaps. We have found that the repopulation of tumour cells is faster during the gap than during the normal days of irradiation. Our model was significantly improved by introducing the duration of the gap into its structure although the overall treatment time has already been taken into account before. The treatment time is prolonged either by increasing the number of fractions (for instance a decrease of the dose per fraction from $2 \mathrm{~Gy}$ to $1.8 \mathrm{~Gy}$ in order to reduce the early normal tissue radiation damage)$\lambda / \alpha=0.36 \mathrm{~Gy} /$ day is lost, or by the break in treatment. When the treatment is interrupted the drop in TCP is even greater, since for one day prolongation of treatment $\lambda / \alpha+\delta / \alpha=0.67$ Gy is lost due to the proliferation of tumour cells. The values of the published estimates of the dose lost due to the prolongation of treatment are in the range of $0.35-0.66 \mathrm{~Gy} /$ day. When the prolongation of treatment is caused by the different dose per fraction the estimates are close to $0.36 \mathrm{~Gy} /$ day [11], while for the changes caused by the treatment gaps the estimates are in the range of $0.5-0.66$ Gy/day [12].

From the practical point of view the conclusion is to reduce as much as possible the number and the duration of treatment interruptions. Unfortunately most of our patients had breaks, some of them more than one. In this group the proportion of the locally controlled patients was very low.

Another important implication is in the way how the treatment time may be reduced in order to minimise the effect of tumour repopulation. The conventional irradiation includes five days of irradiation and a two-day rest for week-ends. The conventional accelerated irradiation (CAIR) was introduced in our institute. The main idea of CAIR was to reduce the treatment time by irradiating during week-ends. This treatment modality proved to be very effective. The preliminary results show that both the local control and the survival was significantly higher in the experimental arm when compared to the conventional treatment. In order to explore the significance of week-ends the third arm was introduced. In this arm patients are not treated during week-ends, but the second fractional dose is given on Tuesdays and Fridays (concomitant boost) to maintain the same overall treatment time as in CAIR. The observation of the concomitant boost arm has not been completed, but the results of the therapy seems to be better than conventional but worse than in the CAIR arm. The results of this randomised prospective study confirm the conclusion drawn from our present retrospective analysis that the repopulation of tumour cells is faster during the days when the tumour is not irradiated.

\section{Acknowledgment}

This research was partly supported by KBN (Polish Committee of Scientific Research) grant no 8T11E03315 for R. Tarnawski and A. Swierniak, and the by fellowship of the Fundation of Polish Science for R. Tarnawski.

The authors thank the anonymous referees whose comments made the essential improvements in the manuscript.

\section{References}

[1] Maciejewski, B., Preuss-Bayer, G. and Trott, K. -R. (1983). The influence of three number of fractions and of overall time on local control and late complications rate in squamous cell carcinoma of the larynx. Int. J. Radiat. Oncol. Biol. Phys., 9 , $321-328$.

[2] Horiot, J.-C., Bontemps, P. van den Bogaert, W. et al. (1997). Accelerated fractionation (AF) compared to conventional fractionation (CF) improves loco-regional control in the radiotherapy of advanced head and neck cancers: results of the EORTC 22851 randomized trial. Radiother. Oncol, 44(2), $111-122$.

[3] Dishe, S., Saunders, M., Barret, A. et al. (1997). A randomised multicentre trial of CHART versus conventional radiotherapy in head and neck cancer. Radiother. Oncol, 44(2), $123-136$.

[4] Trott, K.-R. (1990). Cell repopulation and overall treatment time. Int. J. Radiat. Oncol. Biol. Phys., 19, 1071-1075.

[5] Skladowski, K., Law, M. G., Maciejewski, B. and Steel, G. G. (1994). Planned and unplanned gaps in radiotherapy: the importance of gap position and gap duration. Radiother. Oncol, 30(2), 109-120.

[6] Steel, G. G. (1993). eds. Basic Clinical Radiobiology. London Edward Arnold Publ.

[7] BMDP/386 Dynamic BMDP Statistical Software Inc. (1992). License No. 169-7072.

[8] Bentzen, S. M., Thames, H. D., Travis, E. L., Ang, K. K., van der Schueren, E., Devit, L. and Dixon, D. O. (1989). Direct estimation of latent time for radiation injury in late responding normal tissues: gut, lung, and spinal cord. Int. $J$. Radiat Biol, 55, 27-43. 
[9] Bentzen, S. M., Johansen, L. V., Overgaard, J. and Thames, H. D. (1991). Clinical radiobiology of squamous cell carcinoma of the oropharynx. Int. J. Radiat. Oncol. Biol. Phys., 20, 1197-1206.

[10] Dixon, W. J. (1992). ed. BMDP Statistical Software Manual, 2, Berkeley BMDP Statistical Software Inc.

[11] Hendry, J. H., Roberts, S. A., Slevin, N. J., Keane, T. J., Barton, M. B. and Angren-Cronqvist, A. (1994). Influence of radiotherapy treatment time on control of laryngeal cancer: comparison between centres in Manchester, UK and Toronto, Canada. Radiotherapy Oncology, 31, 14-22.

[12] Withers, H. R., Taylor, J. M. G. and Maciejewski, B. (1988) The hazard of accelerated tumour clonogen repopulation during radiotherapy. Acta Oncol, 27, 131-146. 


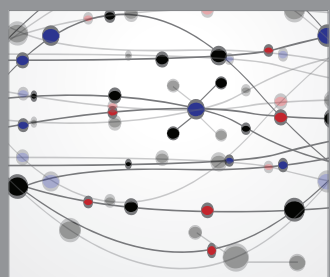

The Scientific World Journal
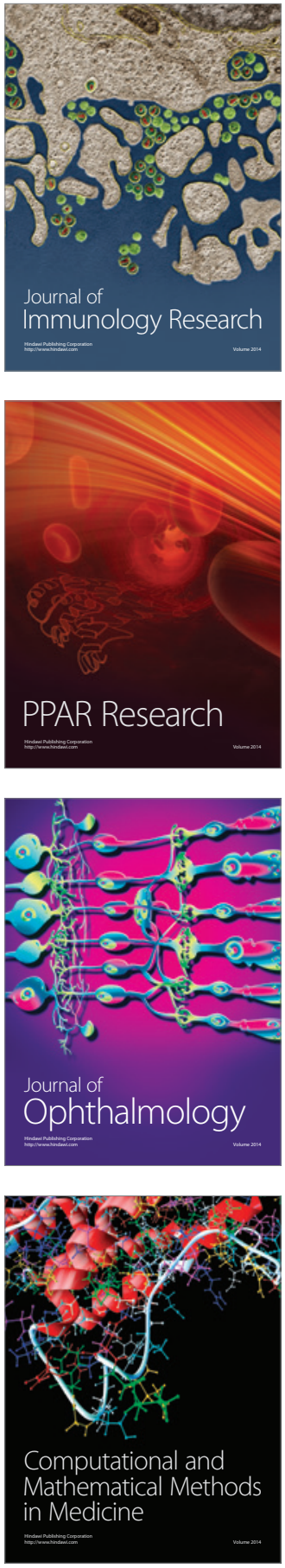

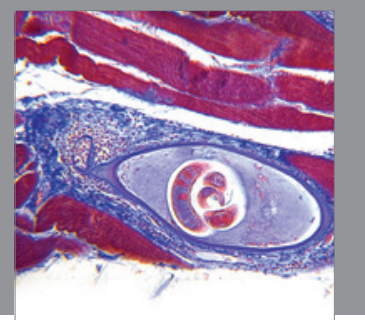

Gastroenterology

Research and Practice
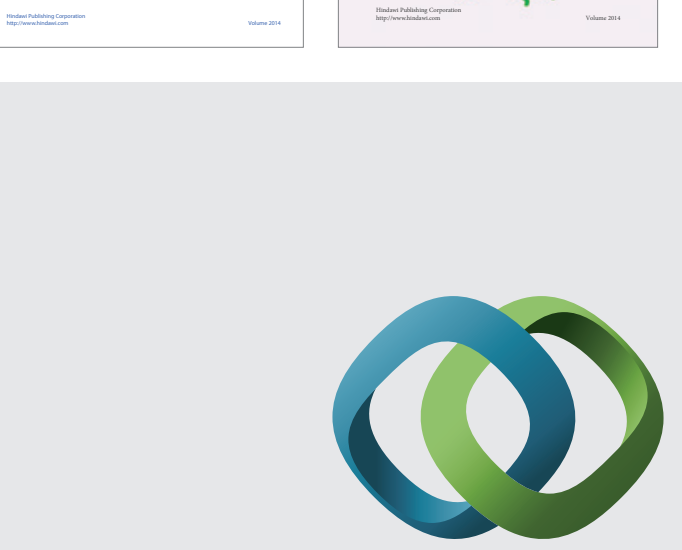

\section{Hindawi}

Submit your manuscripts at

http://www.hindawi.com
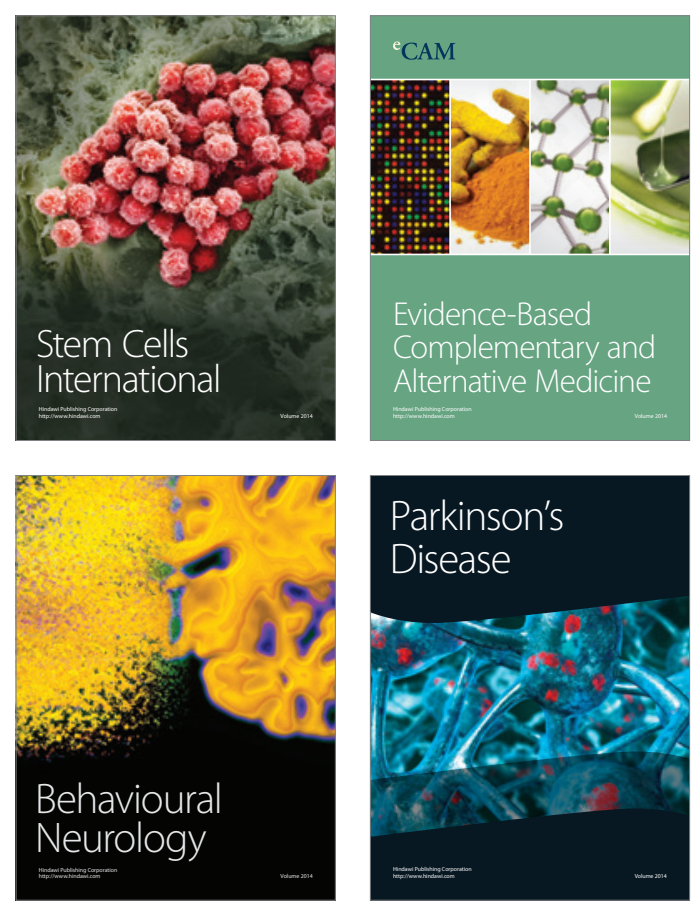

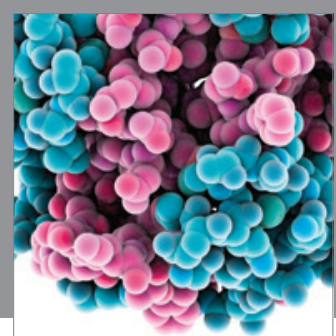

Journal of
Diabetes Research

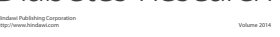

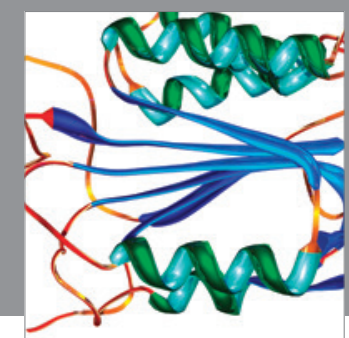

Disease Markers
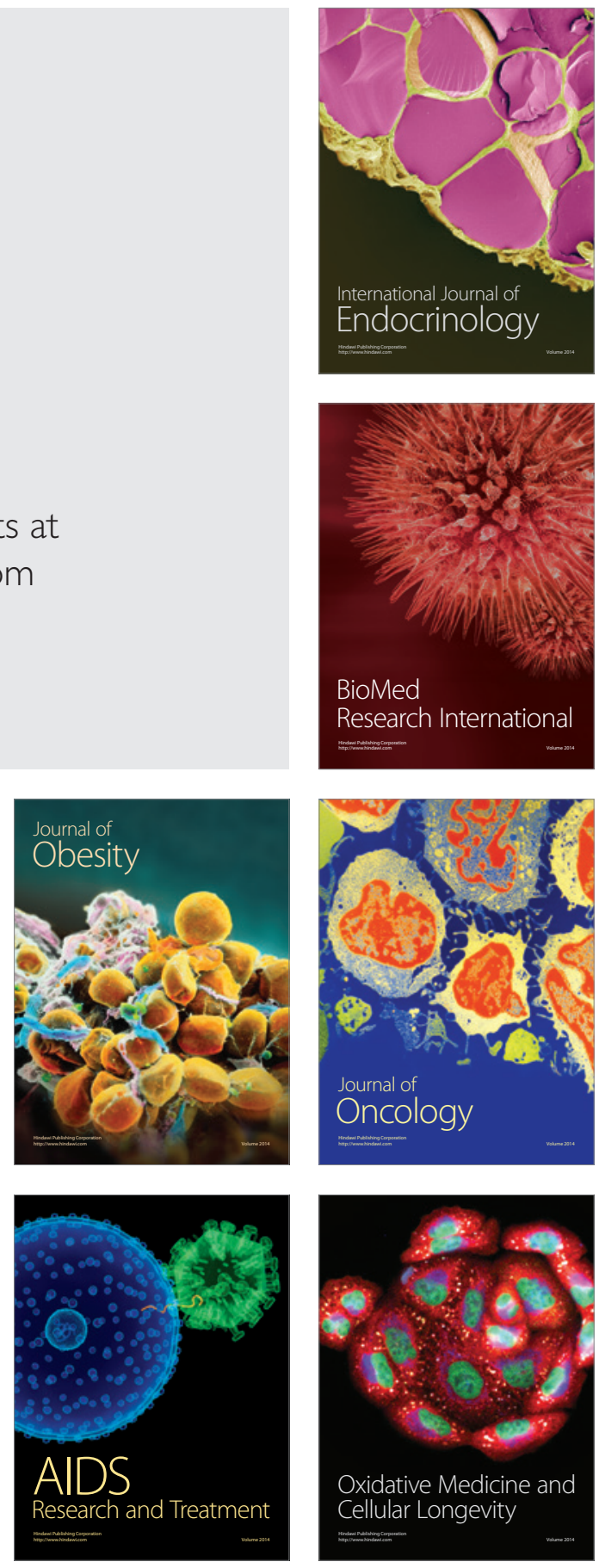